\title{
Utility of CT angiography in cervical spine trauma: analysis of radiation and cost
}

\author{
Waqas Shuaib ${ }^{1}$, Aizaaz Ali Khan ${ }^{2}$, Ajeet Singh Mehta ${ }^{1}$, Arvind Vijayasarathi ${ }^{1}$, Joseph Hidalgo ${ }^{3}$ \\ ${ }^{I}$ Department of Radiology and Imaging Sciences, Emory University Hospital, Atlanta, GA \\ ${ }^{2}$ Department of Emergency Medicine, Grady Memorial Hospital, Atlanta, GA \\ ${ }^{3}$ Department of Surgery, Plaza de la salud, Santo Domingo, DR
}

Received June 26, 2014; Revised July 27, 2014; Accepted August 06, 2014; Published Online August 26, 2014

\section{Original Article}

\begin{abstract}
Purpose: Vertebral artery injuries (VAIs) can be seen in cervical injuries. This investigation was conducted to assess the impact of head and neck computed tomography (CT) angiography (CTA) on planning treatment of vertebral artery injuries, if these tests were ordered appropriately, and to estimate cost and associated exposure to radiation and contrast material. Methods: This retrospective review included all patients who underwent CT of the cervical spine and CTA of the head and neck from March 2011 to October 2012 at a single institution. Patients were divided into two groups, those with and those without cervical spine fracture appreciated on CT of the cervical spine. The frequency of vascular injury on CTA in those with a cervical fracture was assessed. The frequency of vascular injury treatment and modifications owed to a positive CTA of head and neck were also assessed. A study was considered appropriate if it was ordered in accordance with the modified Denver Screening criteria. Effective radiation dose $(\mathrm{mSv})$ was calculated by multiplying dose length product (DLP) from the scanner with the standard conversion coefficient $(\mathrm{k})(\mathrm{k}=0.0021 \mathrm{mSv} / \mathrm{mGy} \mathrm{x} \mathrm{cm})$. Results: In the 387 CTAs of head and neck, a cervical injury was recorded in 128 patients. Twenty CTA scans were correctly ordered for non-spinal indications, and 19 were ordered off protocol. CTA was found positive in 1 patient for whom the imaging was off protocol and 1 for whom the clinical indication was non-cervical. There were 19 positive CTA cases of head and neck, none of which underwent surgical intervention. CTA was positive in 13 of 48 patients who had suffered a C2 fracture; this accounted for 13 of the 19 positive CTA studies $(\mathrm{p}<0.01)$. Estimated fee for CTA was $\$ 3783$, and radiation exposure was $4 \mathrm{mSv}$ with a standard deviation $( \pm 1.3)$. Conclusion: CTA of head and neck ordered off an institutional imaging protocol has a low probability of being positive. Adherence to protocols for CTA of head and neck can reduce costs and decrease unnecessary exposure to radiation and contrast medium.
\end{abstract}

Keywords: CTA; Cerebrovascular Injury; Cervical Trauma

\section{Introduction}

Historically, vertebral artery injuries (VAI) have often gone undiagnosed until devastating consequences present. ${ }^{1-3}$ An early diagnosis allows time to initiate early therapy, including antiplatelet therapy, anticoagulation, and/or surgery if needed. ${ }^{4-7}$ While digital subtraction angiography (DSA) has been regarded as the gold standard in diagnosing VAI, its invasiveness makes it a less desirable imaging modality. ${ }^{8}$

The advent of computed tomography angiography (CTA) has quickly gained momentum in patients who are recognized for screening of VAI. ${ }^{9}$ CT of the head and/or neck, which often is indicated in these patients, can be performed at the same setting. The non-invasive nature, shorter turnaround time, and the lesser amount of contrast used in CTA add to the feasibility of CTA over DSA. ${ }^{8}$ While many studies have credited CTA as an excellent non-invasive alternative to DSA in diagnosing VAI, reports on its appropriate utilization in accordance with the modified Denver Screening Criteria remains an area interest. In our investigation, we assess the application of CTA of head and neck and determine how often imaging protocol was followed, the outcome of those studies, the effect of CTA findings on treatment of cervical fractures, and the outcome of inappropriately ordered studies. 


\section{Methods and Materials}

This study was conducted with institutional review board (IRB) approval and was compliant with the Health Insurance Portability and Accountability Act (HIPPA). The requirement for written informed consent was waived due to the retrospective nature of our study.

We queried the healthcare enterprise data warehouse and the hospital electronic health information system for patients who underwent CT of the cervical spine and CTA of head and neck from March 2011 to October 2012 at a single university-affiliated teaching hospital. CTAs of head and neck were performed on a GE 64-slice scanner (GE VCT Lightspeed, 64-slice; GE Healthcare, Waukesha, WI). Informed consent for IV contrast was obtained. IV contrast (100 cc. Conray 60; Mallinckrodt) was administered via rapid hand infusion prior to scanning for consistent and high quality exams. Two groups were created: 1) Those with cervical spine fracture. 2) Those without cervical spine fracture. In the first group, the following data sets were extracted: site of fracture, presence of VAI, mode of treatment (surgical/nonsurgical), change of treatment (especially, surgical planning), and any neurologic or cerebrovascular problem at latest follow up. In the group without cervical spine fractures, we observed whether CTA of head and neck was positive for VAI. We also assessed the correct use of CTA of head and neck based on the modified Denver Screening Criteria (Table 1) used at our institution. We assessed cost by extracting the charge for CTA of head and neck from our billing system. Radiation exposure and contrast medium used varies from patient to patient. Patient outcome was measured based on review of the patient's medical records at the time of the most recent follow-up.

TABLE 1: Modified Denver screening criteria

\begin{tabular}{|c|c|}
\hline \multicolumn{2}{|c|}{ Denver Modification of Screening Criteria } \\
\hline \multicolumn{2}{|l|}{ Signs/symptoms } \\
\hline $\begin{array}{ll}\text { - } & \text { Arteria } \\
\text { - } & \text { Cervica } \\
\text { - } & \text { Expand } \\
\text { - } & \text { Focal n } \\
\text { - } & \text { Neurol } \\
\text { - } & \text { Iinding } \\
\text { Ischem }\end{array}$ & $\begin{array}{l}\text { emorrhage } \\
\text { g cervit } \\
\text { rological hematoma } \\
\text { ic examination incongruous with CAT scan } \\
\text { stroke on secondary CAT scan }\end{array}$ \\
\hline \multicolumn{2}{|c|}{$\begin{array}{l}\text { - Ischemic stroke on secondary CAT scan } \\
\text { factors }\end{array}$} \\
\hline \multirow{6}{*}{$c$} & rgy transfer mechanism with \\
\hline & Lefort II or III fracture \\
\hline & $\begin{array}{l}\text { Cervical spine fracture patterns: subluxa- } \\
\text { tion, fractures extending into the transverse } \\
\text { foramen, fractures of C1-C3 }\end{array}$ \\
\hline & $\begin{array}{l}\text { Basilar skull fracture with carotid canal } \\
\text { involvement }\end{array}$ \\
\hline & Diffuse axonal injury with GCS $=6$ \\
\hline & Near hanging with anoxic brain injury \\
\hline
\end{tabular}

\section{Estimation of Radiation Dose}

The CT scanner recorded dose length product (DLP) to calculate effective radiation dose. Later, effective radiation dose (mSv) was calculated by multiplying DLP from the scanner with the standard conversion coefficient $(\mathrm{k})(\mathrm{k}=0.0021$ $\mathrm{mSv} / \mathrm{mGy} \mathrm{x} \mathrm{cm})$.

\section{Statistical Analysis}

Statistical analysis was performed using SAS 9.3 (N.C.). Continuous variables were expressed as mean ( \pm standard deviation) and categorical variables as frequency (percentage). Difference between the number of positive CTA exams when the tests were appropriate (for cervical or non-cervical indications) versus the number when they were inappropriate was determined with a chi-square test. The statistical significance was evaluated at alpha $=0.05$.

\section{Results}

A total of 387 patients had a CT of the cervical spine and CTA of head and neck. 128 of these patients had cervical spine fractures (71 men, 57 women), mean age $39 \pm 12$. In $16 \%(21 / 128)$ of the patients VAI was appreciated on CTA. All VAIs were unilateral. Anticoagulation therapy was initiated in 18 patients. Amongst the 18 patients, 9 patients were treated with $325 \mathrm{mg}$ of aspirin twice daily or $81 \mathrm{mg}$ daily, and 4 patients were therapeutically treated with warfarin anticoagulation. None of the VAIs were treated with surgical intervention. Thirteen of the twenty-one patients with VAI underwent surgical therapy for the cervical spine fracture. Average duration of follow-up was 139 days ( \pm 803 days). Irrespective of treatment (surgical vs nonsurgical), all patients with VAI were neurologically intact with no evidence of sequelae from the cerebrovascular injury at their last medical follow-up.

In 48 cases with $\mathrm{C} 2$ fractures, $27 \%(13 / 48)$ had a positive CTA with associated VAI. Six of the twenty-one patients with a VAI had a fracture, which did not include C1, C2, or C3, and 4 of these 21 cases had noncontiguous cervical injuries. In 107 cervical fractures without VAI, 54\% (58/107) included C4-C7. Twenty-two patients in this group had CTA of head and neck ordered off protocol.

TABLE 2: CTA studies of head and neck.

\begin{tabular}{lcc}
\hline \hline Indication & No. (\%) Ordered & No. (\%) Positive \\
\hline Cervical fracture & $89(69 \%)$ & $17(19 \%)$ \\
Non-cervical & $20(16 \%)$ & $1(5 \%)$ \\
Not indicated per protocol & $19(15 \%)$ & $1(5 \%)$ \\
Total No. Ordered & 128 & 19 \\
\hline \hline
\end{tabular}


Of the 128 exams conducted for patients with cervical spine fracture, $16 \%(20 / 128)$ were ordered per protocol for a non-cervical-spine indication and one of the 20 was positive for VAI. Nineteen studies in group 1 were ordered off protocol, and one (5\%) of them was positive for VAI. Other 89 studies were carried out for cervical spine indications per protocol, and 17 (19\%) were positive (Table 2). There was no significant difference in the prevalence of positive CTA findings between the studies ordered for cervical spine indications and those ordered off protocol ( $p=0.10)$, or between all studies ordered per protocol (for cervical or non-cervical indications) and those ordered off protocol $(\mathrm{p}=0.18)$. There was also no significant difference between studies ordered per protocol for cervical indications and those ordered per protocol for non-cervical indications $(\mathrm{p}=0.30)$, or between studies ordered for cervical indications and those ordered for non-cervical indications or off protocol ( $p=0.057$ ). In 259 patients who did not have a cervical fracture, 19 (14\%) with CTA of head and neck were positive for VAI.

Cost of CTA of head and neck was \$3783. Cost for the 19 studies in the fracture group for whom the study was ordered off protocol was $\$ 71,877$. CTA radiation dose per patient was $4 \mathrm{mSv}$ with a standard deviation $( \pm 1.3)$. Contrast-medium load was approximately $100 \mathrm{~mL}$, varying by patient size. There were no contrast medium related anaphylactic reactions in the included patients.

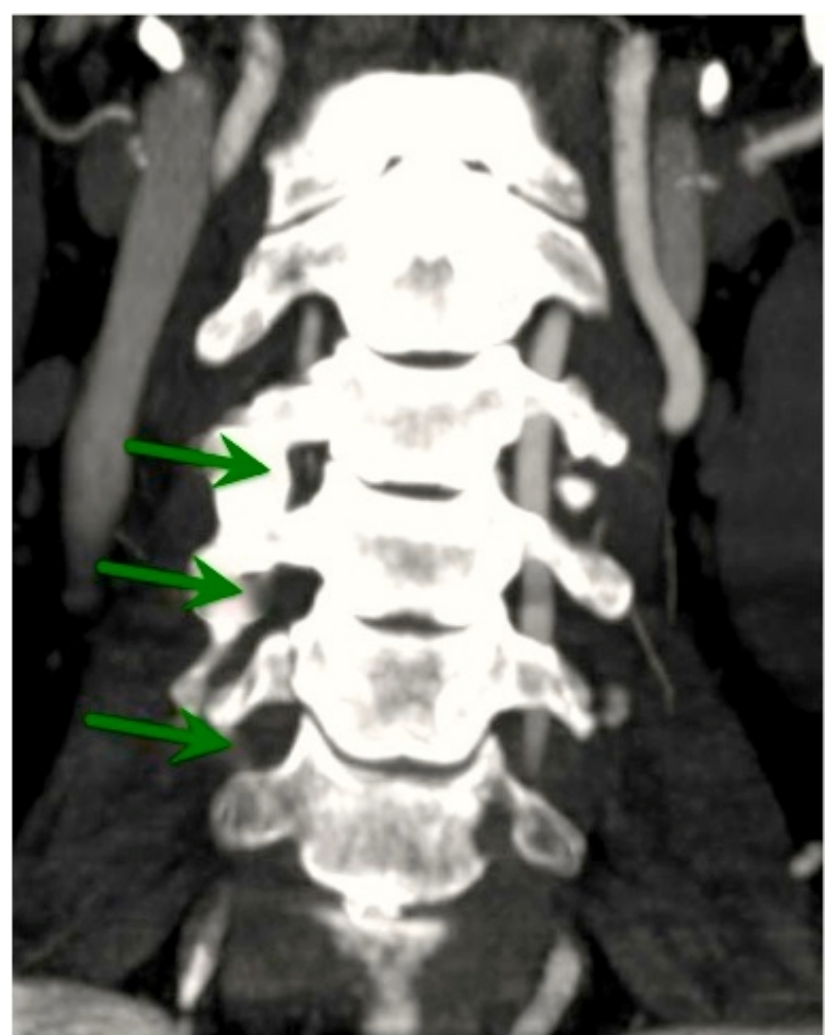

FIG. 1: CT angiography (CTA) of the neck demonstrates a long-segment occlusion (green arrows) of the right vertebral artery from C6 up to base of the skull.

\section{Discussion}

A large number of the scans performed in our investigation were ordered per the modified Denver Screening Criteria either for an indicated cervical fracture pattern (91 patients; $71 \%$ ) or other indicated traumatic injuries (15 patients; $12 \%)$. Nineteen of one hundred and twenty-six CTA scans of head and neck were ordered and executed for fracture patterns off protocol. This issue raises concern in regards to patients receiving unnecessary ionizing radiation. It is well documented that ionizing radiation exposes patients to radiation induced sarcomas ${ }^{10,11}$, and in our investigation a large number of silent VAI went unidentified, as only (5\%) of the studies ordered off protocol were positive. Similarly, studies ordered per protocol for non-cervical-fracture had a low (5\%) probability of being positive, which suggests that, in the event of an absent cervical pathology, the possibility of VAI is low and clinician's should strictly adhere to imaging protocols to ensure proper application of these studies.

Seventeen (19\%) of the studies conducted for patients with cervical fracture were positive for VAI. This is higher than the studies ordered per protocol for non-cervical injuries 1 (5\%), and those ordered off protocol $1(5 \%)$, recommending a benefit in using CTA. While, our data does not show that the criteria used for ordering these studies led to a significant change in identifying VAI. Our data demonstrated a consistent trend towards an increased prevalence of VAI when the study had been ordered for an appropriate cervical fracture; this prevalence was not significantly different from those found with studies ordered for non-cervical-fracture indications or those ordered off protocol.

Contemporary literature reports a correlation of VAI in the presence of upper cervical (C1-C3) fractures. In our investigation, C2 was often the fracture location associated with a VAI, with almost one in four C2 fractures (thirteen patients; 27\%) having a positive finding on CTA of head and neck. These thirteen C2 fractures accounted for more than half of all positive CTA studies of head and neck in this patient population. Contemporary literature questions the conventionally accepted high-risk categories of upper cervical fractures and fractures involving the transverse foramen, and has proposed that VAIs are more common in correlation with displaced cervical injuries amid patients presenting with neurologic deficits. ${ }^{12}$

The need for use of IV contrast in patients undergoing CTA of head and neck is of great clinical concern since contrast nephropathy has been reported in $5 \%$ to $38 \%$ of trauma patients. ${ }^{13}$ Trauma patients often undergo several CT scans and multiple contrast medium loads as part of the initial workup. Additionally, these patients are often hypovolemic on admission, which further potentiates the risk of contrast nephropathy. In our investigation, no patient reported contrast related problems. However, special attention must be 
paid to patient medical history in order to avoid any detrimental anaphylactic reactions that may occur as a result of contrast administration. At our institution, the cost of a CTA of head and neck is $\$ 3783$. The studies that were performed off protocol in our series accounted for more than $\$ 71,877$ in additional expenses. These are potential cost savings, which at a national level can account for millions of dollars.

Our investigation was not without its limitations. The retrospective nature of this investigation does not allow us to establish a definite cause and effect. Also because of its retrospective nature, it is not possible to standardize treatment of either the VAI or the cervical fracture. Our evaluation of medical decision-making in cases where nonoperative measures were taken was limited since this information was not specified in patient notes. Also, laboratory results indicative of renal injury could not be followed unless previously ordered.

\section{Conclusion}

The utility of CTA of head and neck in patients with cervical spine injury depends mainly on the objective for the study. Our investigation suggests that CTA of head and neck is an essential screening tool, which if used properly, can identify VAIs, permitting early pharmacologic treatment. On the other hand, it had minimal effect on decision-making for operative treatment of cervical fractures, and routine CTA of the head and neck for surgical planning is discouraged. Adherence to institutional protocols for CTA studies are encouraged in order to minimize radiation exposure, unnecessary cost, and risk of renal damage related to contrast.

\section{Conflict of interest}

The authors declare that they have no conflicts of interest. The authors alone are responsible for the content and writing of the paper.

\section{References}

1. Scott WW, Sharp S, Figueroa SA, et al. Clinical and radiological outcomes following traumatic Grade 1 and 2 vertebral artery injuries: a 10-year retrospective analysis from a Level 1 trauma center. $J$ Neurosurg 2014; 121:450-6.

2. Desouza RM, Crocker MJ, Haliasos N, et al. Blunt traumatic vertebral artery injury: a clinical review. Eur Spine J2011; 20:1405-16.

3. Biffl WL, Moore EE, Elliott JP, et al. The devastating potential of blunt vertebral arterial injuries. Ann Surg 2000; 231:672-81.
4. Yu W, Binder D, Foster-Barber A, Malek R, et al. Endovascular embolectomy of acute basilar artery occlusion. Neurology 2003; 61:1421-3.

5. Simionato F, Righi C, Silvani P, et al. Emergency endovascular treatment of a life-threatening hemorrhage from traumatic rupture of the left extracranial vertebral artery. Intensive Care Med 1999; 25:1177-9.

6. Shibata T, Ogiichi T, Miyake T, et al. A case of basilar artery occlusion of traumatic vertebral artery dissection successfully managed by endovascular treatment. No Shinkei Geka 2003; 31:311-6.

7. Biffl WL, Ray CE Jr, Moore EE, et al. Treatment-related outcomes from blunt cerebrovascular injuries: importance of routine follow-up arteriography. Ann Surg 2002; 235:699-706; discussion 706-7.

8. Inamasu J, Guiot BH. Vertebral artery injury after blunt cervical trauma: an update. Surg Neurol 2006; 65:238-45; discussion 245-6.

9. Berne JD, Norwood SH, McAuley CE, Villareal DH. Helical computed tomographic angiography: an excellent screening test for blunt cerebrovascular injury. J Trauma 2004; 57:11-7; discussion 17-9.

10. Samartzis D, Nishi N, Hayashi M, et al. Exposure to ionizing radiation and development of bone sarcoma: new insights based on atomic-bomb survivors of Hiroshima and Nagasaki. J Bone Joint Surg Am 2011; 93:1008-15.

11. Shuaib W, Johnson JO, Pande V, et al. Ventriculoperitoneal shunt malfunction: cumulative effect of cost, radiation, and turnaround time on the patient and the health care system. AJR Am J Roentgenol 2014; 202:13-7.

12. Even J, McCullough K, Braly B, et al. Clinical indications for arterial imaging in cervical trauma. Spine 2012; 37:286-91.

13. Trivedi M, Prabhahar T, Preller J. Prevention of contrast induced nephropathy in the critically ill. European Society of Intensive Care Medicine, e-Posters. Vienna 2009. Available from http://poster-consultation.esicm.org/ModuleConsul tationPoster/posterDetail.aspx?intIdPoster=569 